\title{
Composição florística e fitossociologia de macrófitas aquáticas em um banhado continental em Rio Grande, RS, Brasil
}

\author{
Floristic composition and phytosociology of aquatic macrophytes in a continental wetland \\ in Rio Grande, Rio Grande do Sul state, Brazil
}

\author{
Daiane de Sena Kafer ${ }^{1,2}$, Ioni Gonçalves Colares $^{1}$ \& Sonia Marisa Hefler ${ }^{1}$
}

\begin{abstract}
Resumo
Este trabalho teve como objetivo realizar estudo florístico e fitossociológico de macrófitas aquáticas em um banhado continental. Foram distribuídas, sistematicamente, 100 parcelas de $1 \mathrm{~m}^{2}$ (intervalos de $1 \mathrm{~m}$ ), em 10 transectos $(20 \mathrm{~m})$, no Banhado 25. Mensalmente, durante um ano, as plantas foram observadas in situ, coletadas, identificadas e classificadas quanto ao hábito e forma biológica. Para cada espécie registrada foram calculadas cobertura e frequência absoluta e relativa, e o índice de valor de importância (IVI). Foi analisada a similaridade da composição florística (índice de Sorensen) e aplicada regressão linear. Foram levantadas 82 espécies de macrófitas aquáticas vasculares, distribuídas em 33 famílias. Em riqueza de espécies, Cyperaceae (16 táxons), seguida de Poaceae e Asteraceae (12 táxons cada), mostraram maior contribuição. O hábito herbáceo ( $88 \%$ ) e a forma biológica anfíbia (94\%) foram os mais representativos. Scirpus giganteus Kunth apresentou o maior IVI (27,58\%). Maiores índices de cobertura desta espécie demonstraram diminuição significativa na riqueza das espécies $\left(\mathrm{r}^{2}=0,503 ; \mathrm{p}<0,0001\right)$. A alteração na composição florística da margem em relação ao interior do banhado ficou evidenciada pela análise de similaridade, formando dois grupos, possivelmente separados pelo grau de umidade do substrato.
\end{abstract}

Palavras-chave: flora macrofítica, ambientes úmidos, zona costeira, Rio Grande do Sul.

\begin{abstract}
This work aimed to study the floristics and phytosociology of aquatic macrophytes in a continental wetland. A total of, 100 plots, $\left(1 \mathrm{~m}^{2}\right)$ were distributed systematically (intervals of $\left.1 \mathrm{~m}\right)$, in 10 transects $(20 \mathrm{~m})$, in the Banhado 25 area (31 $59^{\prime} 33^{\prime \prime}$; $52^{\circ} 17^{\prime} 32^{\prime \prime}$ ). Monthly collection data over a one-year period included observing plants "in loco", collecting, identifying and classifying the plants according to habit and life form. For each species recorded we calculated absolute and relative frequency and coverage and importance value index (IVI). We analyzed the floristic similarity (Sorensen's index) and applied linear regression. The survey yielded 82 species of vascular aquatic macrophytes, belonging to 33 families. In species richness, Cyperaceae (16 taxa), followed by Poaceae and Asteraceae (12 taxa each), showed greater contribution. The herbaceous habit $(88 \%)$ and amphibious life form (94\%) were the most representative. Scirpus giganteus Kunth had the highest IVI (27.58\%). Higher coverage rates of this species showed a significant decrease in species richness $\left(r^{2}=0.503 ; p<0.0001\right)$. The change in floristic composition between margin and interior of the wetland was evidenced by similarity analysis, forming two groups, possibly separated by humidity.
\end{abstract}

Key word: macrophytic flora, wet environment, coastal zone.

\section{Introdução}

As áreas úmidas são importantes ecossistemas para manutenção da biodiversidade, apresentando grande riqueza de espécies e altos níveis de endemismo (Getzner 2002). Estas áreas são especialmente definidas pela presença de água rasa ou solo saturado, plantas e animais adaptados à vida aquática e acúmulo de matéria orgânica proveniente de vegetais em decomposição, sendo estas as principais características que reúnem ambientes tão diversos (Carvalho \& Osório 2007).

\footnotetext{
${ }^{1}$ Universidade Federal do Rio Grande, Instituto de Ciências Biológicas, Programa de Pós-Graduação em Biologia de Ambientes Aquáticos Continental, Av. Itália, km 8, Campus Carreiros, 96201-900, Rio Grande, RS.

${ }^{2}$ Autor para correspondência: daianesk@yahoo.com.br
} 
A categorização destas áreas pode ser bastante variável. De acordo com Neiff (1997) as áreas úmidas da América do Sul podem ser divididas em duas grandes categorias: 1) grandes áreas úmidas, subdivididas em pantanais e aluviais; 2) áreas úmidas marginais, subdivididas em fluviais, lacustres e litorais. Por outro lado, Barbier et al. (1997) aceitam estas áreas divididas em cinco sistemas: estuarino, marinho, lótico, palustre e lacustre.

Os banhados, representantes de áreas úmidas palustres, apresentam-se como componentes dinâmicos dos ecossistemas, suportando altos níveis de diversidade biológica e de produtividade primária e secundária, além de modular o escoamento das águas, dos nutrientes e outros materiais, provendo importantes componentes para os habitats naturais (Holland et al. 1991). Na região Sul do Brasil os banhados, em sua maioria, estão associados a lagoas costeiras apresentando um grande número de comunidades vegetais que variam principalmente segundo o regime hidrológico, dentre outras características físicas e químicas de cada ambiente (Schwarzbold \& Schäfer 1984).

As macrófitas aquáticas são os vegetais que melhor caracterizam os banhados, pois se ajustam a ambientes alagados (Burger 2010) apresentando uma ampla diversidade de características adaptativas morfofisiológicas (Cook 1996). Nestes ambientes, a vegetação aquática desempenha um importante papel ecológico servindo como fonte de alimento e local de refúgio para diversas espécies de vertebrados e invertebrados, participando da ciclagem e estocagem de nutrientes, da formação de detritos orgânicos e do controle da poluição e da eutrofização artificial (Cabrera \& Fabris 1948; Cook 1996; Esteves 1998).

No entanto, a composição florística e a estrutura da comunidade macrofítica podem ser afetadas por uma série de fatores abióticos, como o teor de nutrientes na água e no sedimento, luminosidade e flutuação dos níveis de água (Maltchik et al. 2004). Destes, o regime hidrológico é um dos fatores mais importantes, pois provoca alterações diretamente na biomassa, composição, produtividade e riqueza (Neiff 1997; Maltchik et al. 2004; Thomaz et al. 2009).

As pesquisas sobre macrófitas aquáticas no Brasil tornaram-se mais frequentes após a década de 90 (Thomaz \& Bini 2003), e em sua grande maioria restringe-se a abordagens florísticas, como por exemplo, os estudos de Bove et al. (2003), Costa et al. (2003), Pivari et al. (2008) e Cervi et al. (2009). Para o Rio Grande do Sul, a obra de maior referência para o estudo de macrófitas aquáticas ainda é de Irgang \&
Gastal Jr. (1996), que catalogaram mais de 300 espécies, levando em conta áreas úmidas de toda a região costeira. Além deste, outros estudos florísticos em âmbito regional foram desenvolvidos, como o levantamento da vegetação costeira do município de Guaíba (Lisbôa \& Gastal 2003), a diversidade de macrófitas em áreas úmidas no município de São Leopoldo (Bertoluci et al. 2004) e o estudo desenvolvido em Palmares do Sul abordando a riqueza das macrófitas aquáticas (Mauhs et al. 2008).

Em Rio Grande, um dos municípios do Estado do Rio Grande do Sul com maior representatividade de ambientes aquáticos (Seeliger et al. 1998), os estudos que envolvem a diversidade das macrófitas, ainda são relativamente escassos (Irgang et al.1984; Cordazzo \& Seeliger 1988; Costa et al. 2003). Sendo assim, o objetivo deste trabalho foi realizar o levantamento florístico e fitossociológico de macrófitas aquáticas em área de um banhado continental, para melhor conhecer a diversidade e estrutura desta comunidade.

\section{Material e Métodos}

\section{Área de estudo}

O presente estudo foi realizado numa área de banhado continental, denominado Banhado 25, localizado no km 32 da BR-392, Rio Grande-Pelotas, distrito de Povo Novo, município do Rio Grande RS (31'59'33'S e 52¹7'32”O) (Fig. 1). Este banhado com uma grande riqueza de espécies nativas é resultado da junção das águas superficiais locais com as águas subterrâneas regionais tanto da Lagoa dos Patos como do Taim e estende-se por cerca de $13 \mathrm{~km}$ no sentido sudeste-nordeste, apresentando uma largura máxima de $1,5 \mathrm{~km}$ (Costa et al. 2003).

Pela grande dimensão, o Banhado 25 acaba assumindo diferentes aspectos fitofisionômicos ao longo de sua extensão, tornando difícil uma caracterização generalizada. Porém, na região do banhado, fragmentada pela BR 392, onde está a área de estudo, pode-se visualizar a representação de pelo menos duas fitofisionomias mais evidentes: a) porções do banhado com domínio de Scirpus giganteus Kunth (Cyperaceae), onde foi realizado o estudo, e, b) porções do banhado com domínio de Cladium jamaicense Crantz (Cyperaceae). Nestas porções encontram-se árvores e arbustos emergentes e esparsos, característicos de ambientes úmidos/ paludosos, áreas de turfeiras, extensões de mata paludosa e campo arenoso adjacente. Deste modo, segundo Maltchik et al. (2004) o banhado se enquadra no sistema palustre, subsistema palustre, do tipo lâmina d' água ausente (ou com área inundada 

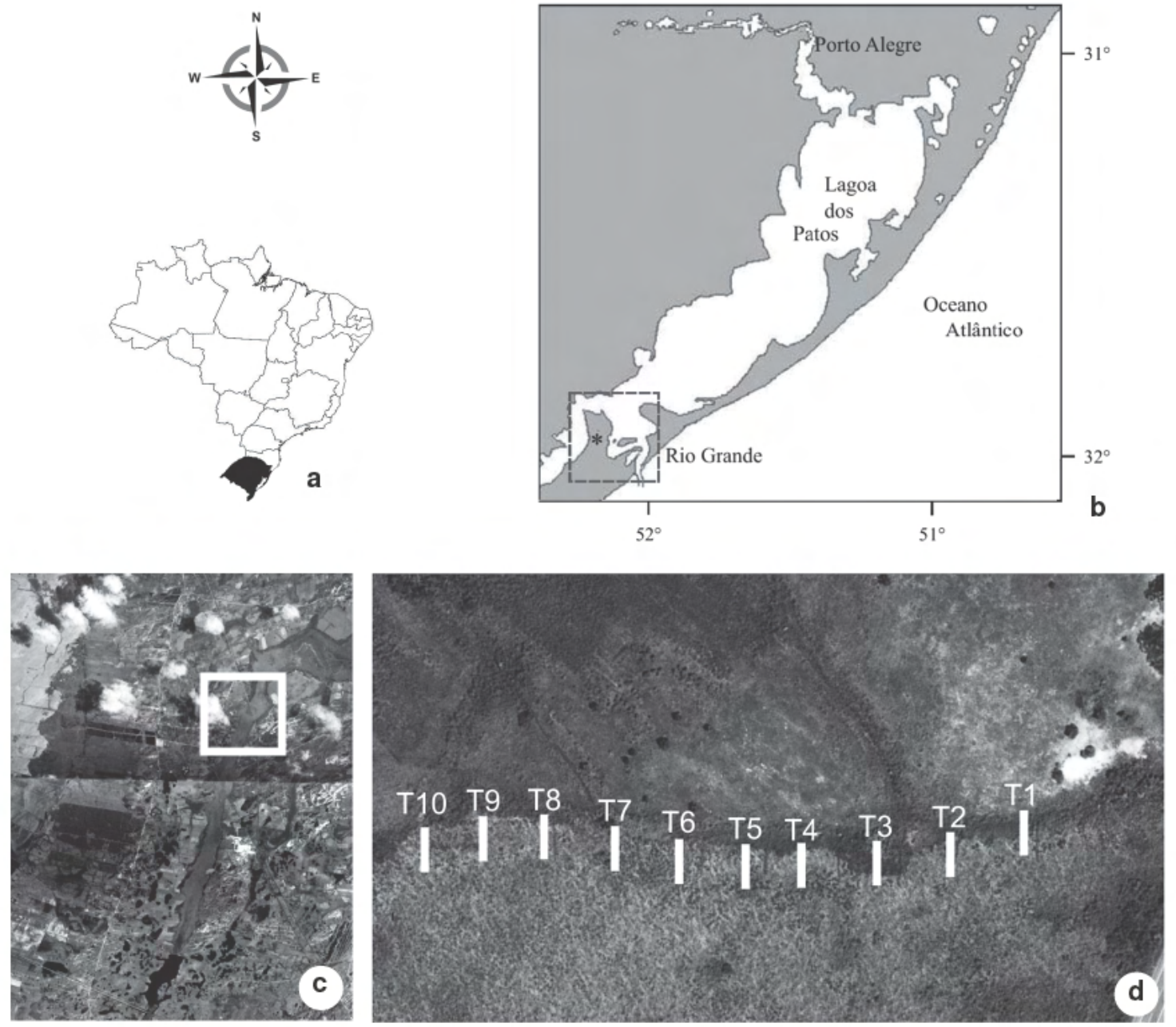

Figura 1 - Área de estudo - a. mapa do Brasil com destaque para o Rio Grande do Sul; b. Planície Costeira do Rio Grande do Sul, localização da cidade do Rio Grande e do Banhado 25; c. imagem de satélite do banhado 25, destaque para área de estudo; d. Banhado 25, mostrando os dez transectos levantados. Fonte: b Freitas et al. (2008), c-d Google Earth (2011). Figure 1 - Study area - a. map of Brazil, especially Rio Grande do Sul; b. Coastal Plain of Rio Grande do Sul, located in Rio Grande and the Banhado 25; c. satellite image of the Banhado 25, particularly the study area; d. Banhado 25, showing the ten transects surveyed. Font: b Freitas et al. (2008), c-d Google Earth (2011).

pelo menos 4 meses do ano) com predominância de indivíduos emergentes.

O clima da região é caracterizado como subtropical úmido, com temperatura média anual de $17^{\circ} \mathrm{C}$, no mês mais quente (janeiro) de $27^{\circ} \mathrm{C}$ e no mais frio (julho) de $8,87^{\circ} \mathrm{C}$ (Krusche et al. 2002). A precipitação pluviométrica varia entre 58,31 e $147,68 \mathrm{~mm}$, sendo janeiro e julho, os meses de menor e maior precipitação, respectivamente e os ventos de nordeste dominam durante a maior parte do ano, variando as velocidades médias entre 2,62 e 4,28 m/s (Krusche et al. 2002).

\section{Coleta e análise de dados}

O levantamento florístico e fitossociológico das macrófitas aquáticas foi realizado entre dezembro de 2008 e janeiro de 2010 por meio de expedições mensais a área de estudo. Neste período foram realizadas coletas de material botânico, fotos e observações em campo.

Para a amostragem foram estabelecidos dez transectos permanentes de $20 \mathrm{~m}$, sentido margemcentro do banhado, distanciados entre si cerca de $30 \mathrm{~m}$ (Fig. 1d). O ponto inicial de cada transecto foi estabelecido pela margem do banhado que apresentou 
elevação topográfica (barranco) delimitando a área de campo adjacente. Para cada transecto foram delimitadas dez parcelas de $1 \mathrm{~m}^{2}$, alternadas sistematicamente, com espaçamento de $1 \mathrm{~m}$ entre elas, totalizando 100 unidades amostrais (UA's). Em cada parcela foi avaliada a cobertura ocupada pelas espécies de macrófitas aquáticas baseada na escala de Domin-Krajina (BraunBlanquet 1979, adaptado para este estudo): "1" cobertura de até $10 \%$ da área da parcela, " 2 " - cobertura entre 11 e $20 \%$, "3" - cobertura entre 21 e $40 \%$, "4" cobertura entre 41 e $60 \%$, "5" - cobertura entre 61 e $80 \%$ e "6" = cobertura entre 81 e $100 \%$. O enquadramento taxonômico segue o proposto pelo APG III (2009) e a nomenclatura correta dos nomes está de acordo com Tropicos (2010) e Forzza et al. (2010). O material coletado está depositado no HURG (Herbário da Universidade do Rio Grande, Instituto de Ciências Biológicas, Universidade Federal do Rio Grande).

As espécies foram classificadas quanto ao hábito, em herbáceas, arbustivas, lianescentes ou arbóreas e quanto à forma biológica, nas categorias propostas por Irgang \& Gastal Jr. (1996).

A suficiência amostral foi estimada em uma curva de táxons por área amostrada, ajustada por regressão logarítmica, construída em planilha eletrônica. Para cada espécie registrada no levantamento foi calculada a cobertura absoluta $(\mathrm{Ca})$, frequência absoluta $(\mathrm{Fa})$ cobertura relativa $(\mathrm{Cr})$, frequência relativa $(\mathrm{Fr})$ e o índice de valor de importância (IVI) (Müller-Dombois \& Ellenberg 1974). A partir dos cálculos dos parâmetros fitossociológicos a espécie com maior dominância teve sua cobertura correlacionada com a riqueza de espécies encontrada em cada unidade amostral. Para esta análise foi realizada uma regressão linear simples utilizando a cobertura total da espécie e a riqueza encontrada em cada UA. As espécies que apresentaram frequência absoluta menor ou igual a $5 \%$ foram consideradas raras neste estudo.

A análise de agrupamento foi empregada na avaliação da similaridade entre as 100 parcelas. Para esta análise foi utilizada a matriz de presença e ausência de espécies, a partir da qual foi realizada a análise de agrupamentos pelo método UPGMA, considerando como medida de similaridade o Índice de Sorensen (Müller-Dombois \& Ellenberg 1974). Todos os cálculos estatísticos foram realizados pelo software PAST (Hammer et al. 2001).

\section{Resultados}

O levantamento florístico da área estudada revelou 82 espécies de macrófitas aquáticas vasculares, distribuídas em 63 gêneros e 33 famílias
(Tab. 1), sendo que $60 \%$ das famílias e $81 \%$ dos gêneros são monoespecíficos. Quatro famílias pertencem às Pteridófitas sensu lato (Equisetaceae, Pteridaceae, Osmundaceae e Thelipteridaceae) e as restantes às Angiospermas (Tab. 1). A família mais representativa, em termos de riqueza de espécies foi Cyperaceae (16 espécies), seguida de Asteraceae (12) e Poaceae (12) (Fig. 2). Essas três famílias compreenderam $49 \%$ das espécies levantadas.

A curva do número cumulativo de espécies em função da área levantada mostrou uma tendência à estabilização a partir da $80^{\circ}$ unidade amostral $\left(r^{2}=0,97 ; y=18,5 \ln (x)-1,1\right)$, quando $96 \%$ das espécies registradas já estavam amostradas (Fig. 3).

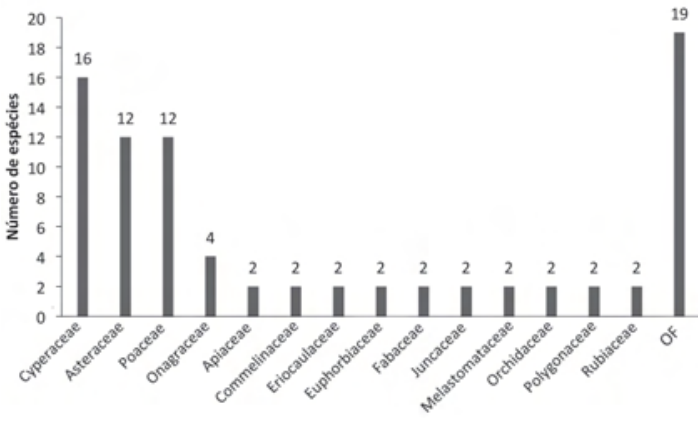

Figura 2 - Riqueza específica das famílias botânicas encontradas no Banhado 25. OF (outras famílias) = soma da riqueza específica das famílias com apenas uma espécie. Figure 2 - Species richness of plant families found in the Banhado 25. OF (other families) = sum of species richness of families with one species.

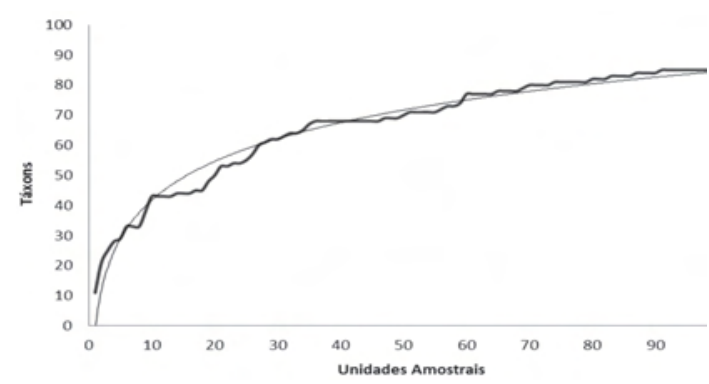

Figura 3 - Curva do número cumulativo de espécies por área para o levantamento fitossociológico, ajustada com curva de regressão logarítmica.

Figure 3 - Curve of cumulative number of species with area for the phytosociological survey, adjusted with logarithmic regression curve. 
Tabela 1 - Lista de espécies com suas respectivas famílias, hábito, forma biológica e parâmetros fitossociológicos registradas em 100 unidades amostrais no Banhado 25. Hábito: $\mathrm{H}=$ herbáceo, $\mathrm{A}=$ arbustivo, $\mathrm{Ar}=\operatorname{arbóreo,~} \mathrm{L}=$ lianas. Forma biológica: $\mathrm{A}$ = anfíbia, $\mathrm{E}=$ emergente. $\mathrm{Uai}=$ números de unidades amostrais onde a espécie "ij" ocorre, $\mathrm{Ca}=$ cobertura absoluta, $\mathrm{Fa}=$ frequência absoluta, $\mathrm{Cr}=$ cobertura relativa, $\mathrm{Fr}=$ frequência relativa, $\mathrm{IVI}=$ índice de valor de importância. Negrito = 14 espécies com maior IVI.

Table 1 - List of species with their respective family, habit, biological form and phytosociological parameters found in 100 sampling units in Banhado 25. Habit: $\mathrm{H}=$ herb, shrub $=\mathrm{A}, \mathrm{Ar}=$ arboreal, $\mathrm{L}=$ Liana. Biological form: $\mathrm{A}=$ amphibian, $\mathrm{E}=$ emergent. Uai $=$ number of plots where the species " $\mathrm{i}$ " occurs, $\mathrm{Ca}=$ absolute cover, $\mathrm{Fa}=$ absolute frequency, $\mathrm{Cr}=$ relative coverage, $\mathrm{Fr}=\mathrm{relative}$ frequency, IVI = Importance Value Index. Bold $=14$ species with the highest IVI.

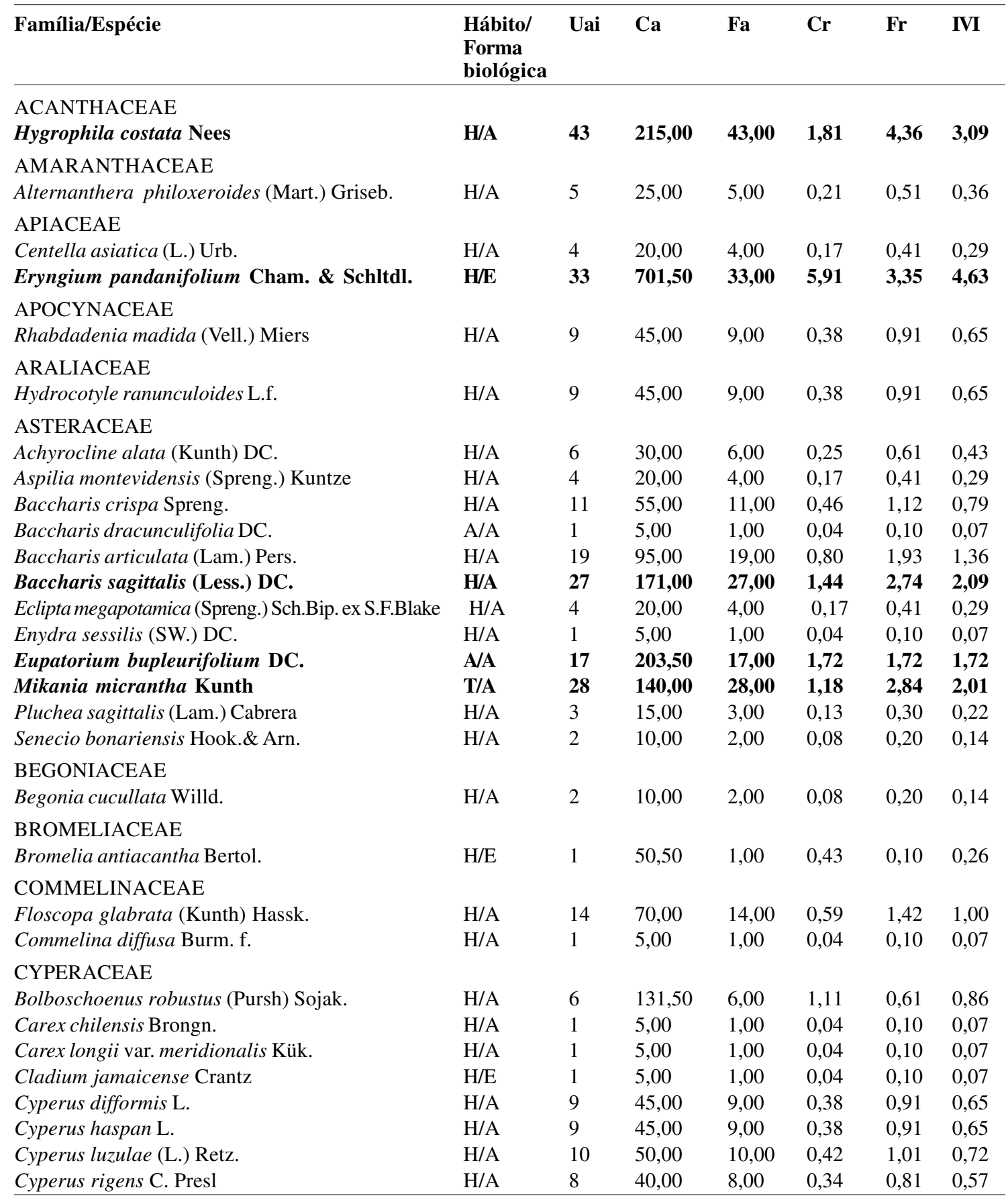




\begin{tabular}{|c|c|c|c|c|c|c|c|}
\hline Família/Espécie & $\begin{array}{l}\text { Hábito/ } \\
\text { Forma } \\
\text { biológica }\end{array}$ & Uai & $\mathbf{C a}$ & $\mathbf{F a}$ & $\mathbf{C r}$ & Fr & IVI \\
\hline Eleocharis acutangula (Roxb.) Schult. & $\mathrm{H} / \mathrm{A}$ & 10 & 238,00 & 10,00 & 2,01 & 1,01 & 1,51 \\
\hline Eleocharis interstincta (Vahl) Roem. \& Schult. & H/A & 7 & 35,00 & 7,00 & 0,30 & 0,71 & 0,50 \\
\hline Eleocharis nudipes Palla & $\mathrm{H} / \mathrm{A}$ & 3 & 15,00 & 3,00 & 0,13 & 0,30 & 0,22 \\
\hline Pycreus polystachyos (Rottb.) P.Beauv. & H/A & 3 & 15,00 & 3,00 & 0,13 & 0,30 & 0,22 \\
\hline Rhynchospora marisculus Lindl. \& Nees & $\mathrm{H} / \mathrm{A}$ & 8 & 126,50 & 8,00 & 1,07 & 0,81 & 0,94 \\
\hline Scirpus giganteus Kunth & $\mathbf{H} / \mathbf{E}$ & 88 & 5486,50 & 88,00 & 46,25 & 8,92 & 27,59 \\
\hline Scleria malaleuca Rchb. ex Schltdl. \& Cham. & $\mathrm{H} / \mathrm{A}$ & 2 & 10,00 & 2,00 & 0,08 & 0,20 & 0,14 \\
\hline Scleria hirtella SW. & $\mathrm{H} / \mathrm{A}$ & 9 & 127,50 & 9,00 & 1,07 & 0,91 & 0,99 \\
\hline $\begin{array}{l}\text { EQUISETACEAE } \\
\text { Equisetum giganteum } \mathrm{L} \text {. }\end{array}$ & H/A & 9 & 45,00 & 9,00 & 0,38 & 0,91 & 0,65 \\
\hline ERIOCAULACEAE & & & & & & & \\
\hline Eriocaulon magnificum Ruhland & $\mathrm{H} / \mathrm{E}$ & 11 & 238,00 & 11,00 & 2,01 & 1,12 & 1,56 \\
\hline EUPHORBIACEAE & & & & & & & \\
\hline Caperonia linearifolia A.St.-Hil. & H/A & 3 & 15,00 & 3,00 & 0,13 & 0,30 & 0,22 \\
\hline Sapium glandulosum (L.) Morong & $\mathrm{Ar} / \mathrm{A}$ & 3 & 15,00 & 3,00 & 0,13 & 0,30 & 0,22 \\
\hline FABACEAE & & & & & & & \\
\hline Desmodium incanum DC. & $\mathrm{T} / \mathrm{A}$ & 6 & 30,00 & 6,00 & 0,25 & 0,61 & 0,43 \\
\hline Vigna luteola (Jacq.) Benth. & T/A & 35 & 175,00 & 35,00 & 1,48 & 3,55 & 2,51 \\
\hline $\begin{array}{l}\text { GESNERIACEAE } \\
\text { Sinningia elatior (Kunth) Chautems }\end{array}$ & H/A & 1 & 5,00 & 1,00 & 0,04 & 0,10 & 0,07 \\
\hline IRIDACEAE & & & & & & & \\
\hline Cypella coelestis (Lehm.) Diels & $\mathrm{H} / \mathrm{A}$ & 1 & 5,00 & 1,00 & 0,04 & 0,10 & 0,07 \\
\hline JUNCACEAE & & & & & & & \\
\hline Juncus scirpoides Lam. & $\mathrm{H} / \mathrm{A}$ & 3 & 15,00 & 3,00 & 0,13 & 0,30 & 0,22 \\
\hline Juncus microcephalus Kunth & $\mathrm{H} / \mathrm{A}$ & 3 & 15,00 & 3,00 & 0,13 & 0,30 & 0,22 \\
\hline LAMIACEAE & & & & & & & \\
\hline Hyptis fasciculata Benth. & H/A & 40 & 200,00 & 40,00 & 1,69 & 4,06 & 2,87 \\
\hline $\begin{array}{l}\text { LYTHRACEAE } \\
\text { Cuphea carthagenensis (Jacq.) J. Macbr. }\end{array}$ & $\mathrm{H} / \mathrm{A}$ & 6 & 30,00 & 6,00 & 0,25 & 0,61 & 0,43 \\
\hline MALVACEAE & & & & & & & \\
\hline Pavonia distinguenda A. St.-Hil. \& Naudin & $\mathrm{H} / \mathrm{A}$ & 5 & 25,00 & 5,00 & 0,21 & 0,51 & 0,36 \\
\hline $\begin{array}{l}\text { MAYACACEAE } \\
\text { Mayaca sellowiana Kunth }\end{array}$ & $\mathrm{H} / \mathrm{A}$ & 2 & 10,00 & 2,00 & 0,08 & 0,20 & 0,14 \\
\hline MELASTOMATACEAE & & & & & & & \\
\hline Tibouchina asperior (Cham.) Cogn. & HA & 40 & 444,50 & 40,00 & 3,75 & 4,06 & 3,90 \\
\hline Tibouchina gracilis (Bonpl.) Cogn. & $\mathrm{H} / \mathrm{A}$ & 7 & 35,00 & 7,00 & 0,30 & 0,71 & 0,50 \\
\hline ONAGRACEAE & & & & & & & \\
\hline Ludwigia grandiflora (Michx.) Greuter \& Burdet & $\mathrm{H} / \mathrm{A}$ & 6 & 30,00 & 6,00 & 0,25 & 0,61 & 0,43 \\
\hline Ludwigia longifolia (DC.) H.Hara & $\mathrm{H} / \mathrm{A}$ & 3 & 15,00 & 3,00 & 0,13 & 0,30 & 0,22 \\
\hline Ludwigia multinervia (Hook. \& Arn.) Ramamoorthy & $\mathbf{H} / \mathbf{A}$ & 42 & 231,00 & 42,00 & 1,95 & 4,26 & 3,10 \\
\hline Ludwigia tomentosa (Cambess.) H.Hara & $\mathrm{H} / \mathrm{A}$ & 16 & 80,00 & 16,00 & 0,67 & 1,62 & 1,15 \\
\hline Habenaria .brachyphyton Schultr. & $\mathrm{H} / \mathrm{A}$ & 1 & 5,00 & 1,00 & 0,04 & 0,10 & 0,07 \\
\hline Habenaria repens Nutt. & $\mathrm{H} / \mathrm{A}$ & 1 & 5,00 & 1,00 & 0,04 & 0,10 & 0,07 \\
\hline $\begin{array}{l}\text { OSMUNDACEAE } \\
\text { Osmunda regalis L. }\end{array}$ & $\mathrm{H} / \mathrm{A}$ & 3 & 15,00 & 3,00 & 0,13 & 0,30 & 0,22 \\
\hline POACEAE & & & & & & & \\
\hline Andropogon sp. & $\mathrm{H} / \mathrm{A}$ & 11 & 127,00 & 11,00 & 1,07 & 1,12 & 1,09 \\
\hline Briza calotheca (Trin.) Hack. & H/A & 11 & 65,50 & 11,00 & $\mathbf{0 , 5 5}$ & 1,12 & $\mathbf{0 , 8 3}$ \\
\hline
\end{tabular}




\begin{tabular}{|c|c|c|c|c|c|c|c|}
\hline Família/Espécie & $\begin{array}{l}\text { Hábito/ } \\
\text { Forma } \\
\text { biológica }\end{array}$ & Uai & $\mathbf{C a}$ & $\mathbf{F a}$ & $\mathrm{Cr}$ & Fr & IVI \\
\hline $\begin{array}{l}\text { Dicanthelium sabulorum (Lam.) } \\
\text { Gould \& C.A. Clarck }\end{array}$ & $\mathbf{H} / \mathbf{A}$ & 55 & 332,00 & 55,00 & 2,80 & 5,58 & 4,19 \\
\hline Erianthus trinii (Hack.) Hack. & H/A & 7 & 35,00 & 7,00 & 0,30 & 0,71 & 0,50 \\
\hline Eriochrysis cayennensis P.Beauv. & $\mathrm{H} / \mathrm{A}$ & 1 & 5,00 & 1,00 & 0,04 & 0,10 & 0,07 \\
\hline Hypoginum virgatum (Desv) Dandy & H/A & 3 & 15,00 & 3,00 & 0,13 & 0,30 & 0,22 \\
\hline Ischaemum minus J.Presl & $\mathrm{H} / \mathrm{A}$ & 9 & 45,00 & 9,00 & 0,38 & 0,91 & 0,65 \\
\hline Leersia hexandra Sw. & H/A & 61 & $\mathbf{3 1 5 , 5 0}$ & 61,00 & 2,66 & 6,19 & 4,42 \\
\hline Panicum aquaticum Poir. & $\mathrm{H} / \mathrm{A}$ & 2 & 10,00 & 2,00 & 0,08 & 0,20 & 0,14 \\
\hline Paspalum urvillei Steud. & $\mathrm{H} / \mathrm{A}$ & 2 & 10,00 & 2,00 & 0,08 & 0,20 & 0,14 \\
\hline $\begin{array}{l}\text { Schyzachyrium microstachyum (Desv. ex Ham.) } \\
\text { Roseng., B.R. Arrill. \& Izag. }\end{array}$ & $\mathrm{H} / \mathrm{A}$ & 2 & 10,00 & 2,00 & 0,08 & 0,20 & 0,14 \\
\hline Sporobulos sp. & $\mathrm{H} / \mathrm{A}$ & 1 & 5,00 & 1,00 & 0,04 & 0,10 & 0,07 \\
\hline POLYGONACEAE & & & & & & & \\
\hline Polygonum acuminatum Kunth & $\mathrm{H} / \mathrm{A}$ & 5 & 25,00 & 5,00 & 0,21 & 0,51 & 0,36 \\
\hline Polygonum meisnerianum Cham. & $\mathrm{H} / \mathrm{A}$ & 13 & 65,00 & 13,00 & 0,55 & 1,32 & 0,93 \\
\hline PTERIDACEA & & & & & & & \\
\hline Achrostichum danaeifolium Langsd. \& Fish. & $\mathbf{H} / \mathbf{E}$ & 61 & 397,00 & 61,00 & 3,35 & 6,19 & 4,77 \\
\hline SCROPHULARIACEAE & & & & & & & \\
\hline Buddleja thyrsoides Lam. & $\mathrm{A} / \mathrm{A}$ & 1 & 5,00 & 1,00 & 0,04 & 0,10 & 0,07 \\
\hline Galium hypocarpium (L.) Endl. ex Griseb. & T/A & 1 & 5,00 & 1,00 & 0,04 & 0,10 & 0,07 \\
\hline Galium equisetoides (Cham. \& Schltdl.) Standl. & T/A & 20 & 100,00 & 20,00 & 0,84 & 2,03 & 1,44 \\
\hline THELIPTERIDACEAE & & & & & & & \\
\hline Thelypteris interrupta (Willd.) K. Iwats. & $\mathrm{H} / \mathrm{A}$ & 13 & 65,00 & 13,00 & 0,55 & 1,32 & 0,93 \\
\hline URTICACEAE & & & & & & & \\
\hline Boehmeria cylindrica (L.) Sw. & $\mathbf{H} / \mathbf{A}$ & 33 & 165,00 & 33,00 & 1,39 & 3,35 & 2,37 \\
\hline XYRIDACEAE & & & & & & & \\
\hline Xyris jupicai Rich. & H/A & 2 & 10,00 & 2,00 & 0,08 & 0,20 & 0,14 \\
\hline
\end{tabular}

Na análise fitossociológica, as 14 espécies com maior valor de importância somaram 77,38\% do valor de cobertura relativa e $61,15 \%$ do valor de frequência relativa da área estudada (Tab. 1, Fig. 4). A espécie mais importante foi Scirpus giganteus com 46,25\% de cobertura relativa e $8,92 \%$ de frequência relativa, apresentando um valor de importância de 27,58\%. A seguir encontramos Achrostichum danaeifolium, Eryngium pandanifolium, Leersia hexandra, Dicanthelium sabulorum e Tibouchina asperior, que apresentaram valores de importância, entre 3,90\% e 4,77\% (Fig. 4), que embora frequentes na área do estudo, apresentaram baixo valor de cobertura.

As espécies raras, que tiveram ocorrência em menos de cinco unidades amostrais, foram representadas por quase a metade das espécies encontradas no estudo (36 espécies), correspondendo juntas apenas 5,43\% do valor de importância total (Fig. 4). O hábito herbáceo foi o mais frequente, com $88 \%$, seguido do lianescente com $6 \%$, arbustivo com
5\% e arbóreo representado apenas por Sapium glandulosum (L.) Morong (1\%) (Fig. 5). Entre as formas biológicas, foram registradas apenas espécies anfíbias e emergentes, sendo a primeira mais representativa (94\%) (Fig. 5).

Não houve alteração na taxa de cobertura em relação à composição das espécies ao longo do estudo, permanecendo $S$. giganteus dominante na maioria das parcelas. Do mesmo modo, outras espécies (Eriocaulon magnificum, Eryngium pandanifolium, Tibouchina asperior) que detinham a maior taxa de cobertura em algumas parcelas, também mantiveram seus índices ao longo do período estudado.

A regressão linear (Fig. 6) revelou que à medida que a cobertura de $S$. giganteus aumenta, menor é o número de espécies presentes nas parcelas $\left(\mathrm{r}^{2}=0,50 ; \mathrm{y}=0,07 \mathrm{x}+13,5 ; \mathrm{p}<0,0001\right)$. Com relação à similaridade florística entre as parcelas, podemos observar a formação de dois grupos distintos (Fig. 7), quando considerado o corte a 


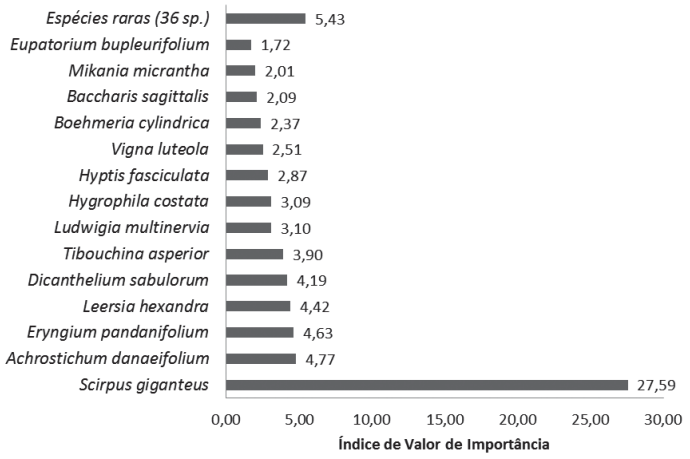

Figura 4 - Índice de valor de importância das 14 espécies com maiores valores. Espécies raras = soma dos IVI das 36 espécies que ocorreram em menos de $5 \%$ das unidades amostrais levantadas.

Figure 4 - Index of importance value of 14 species with higher values. Rare species $=$ sum of IVI of 36 species that occur in less than $5 \%$ of sample units raised.

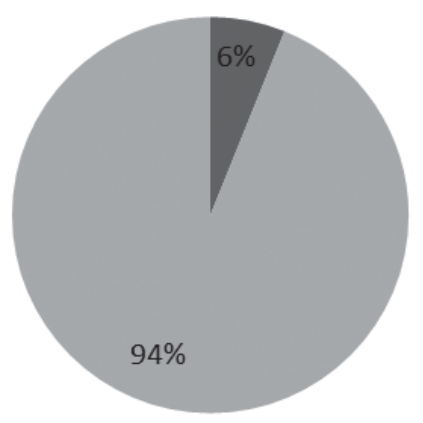

Espécies

emergentes

Espécies anfíbias

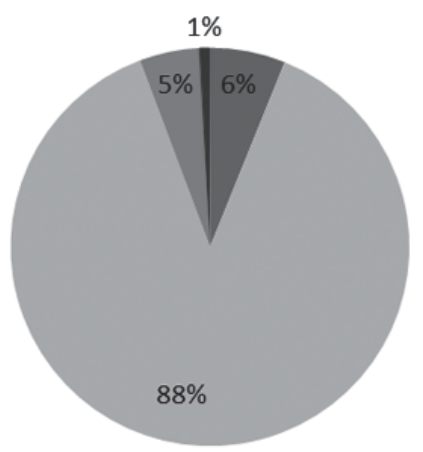

- Lianas

- Herbáceas

Arbustivas

Arbóreas

Figura 5 - Representação gráfica da forma biológica e hábito das espécies levantadas no Banhado 25. a. forma biológica; b. hábito.

Figure 5 - Graphical representation of biological form and habit of the species raised in Banhado 25. a. biological form; b. habit.

cerca de $65 \%$ de similaridade. Estes grupos, aqui denominados A e B, mostraram diferenças na composição florística entre as parcelas marginais em relação às do interior do banhado, sendo a ocorrência de espécies raras a maior contribuição para a definição deste agrupamento.

O grupo A, representado pelo conjunto de parcelas levantadas a partir do meio dos transectos em direção ao centro do banhado (P6-P10), apresentou maior cobertura de $S$. giganteus $(66,12 \%)$. Neste agrupamento se evidenciou a ocorrência de espécies restritas a estas parcelas, como Alternanthera philoxeroides, Polygonum acuminatum, Rhabdadenia madida, Ludwigia longifolia, e algumas espécies raras para o local estudado, como Caperonia linearifolia, Begonia cucullata, Carex chilensis, Cladium jamaicense, entre outras.

O grupo B, representado pelo conjunto de parcelas da margem do banhado até o meio dos transectos (P1-P5), apresentou uma redução na cobertura pelo $S$. giganteus $(33,87 \%)$, em relação ao grupo anterior. Neste agrupamento ocorreu maior número de espécies restritas a esta área, quando comparado ao grupo A, sendo muitas destas raras para o local de estudo, como Cypella coelestis, Enydra sessilis, Galium hypocarpium, Habenaria brachyphyton, Habenaria repens, Sinningia elatior, Eriochrysis cayennensis e Carex longii var. meridionalis. Algumas das espécies raras, pelo seu porte ou arquitetura, passam a compor grande parte da cobertura das parcelas onde estão presentes, como Bromelia antiacantha, Baccharis dracunculifolia e Buddleja thyrsoides.

a

\section{Discussão}

O número de espécies de macrófitas aquáticas encontrado na área amostrada do Banhado 25 (82 espécies) pode ser considerado representativo tendo-se como parâmetro o tamanho da área levantada e os trabalhos de Matias et al. (2003) com 45 espécies, Kita \& Souza (2003) com 89 espécies, Pivari et al. (2008) com 56 espécies, Mauhs et al. (2008) com 18 espécies e Moura-Júnior et al. (2009) com 48 espécies registradas. No entanto, esse número foi inferior ao encontrado por Spellmeir $e t$ al. (2009), cuja amostragem compreendeu toda extensão de um banhado, em Estrela-RS (145 espécies) e por Cervi et al. (2009) que levantaram os principais rios, brejos e lagoas que ocorrem em General Carneiro-PR (117 espécies). Deve-se considerar ainda que as áreas amostradas foram maiores em relação ao presente estudo. 
A ocorrência de famílias e gêneros representados por apenas uma espécie parece ser comum em levantamentos de macrófitas aquáticas como também constatado nos estudos de Amato et al. (2007) e Rocha et al. (2007). Das famílias monoespecíficas, destacam-se especialmente Acanthaceae (Hygrophila costata), Amaranthaceae (Alternanthera philoxeroides), Apocynaceae (Rhabdadenia madida), Begoniaceae (Begonia cucullata), Equisetaceae (Equisetum giganteum), Lythraceae (Cuphea carthagenensis) e Mayacaceae (Mayaca sellowiana), bastante comuns em levantamentos da flora macrofítica aquática nas regiões austrais do Brasil (Irgang et al. 1984; Pott et al. 1989; Irgang \& Gastal Jr.1996; Amaral et al. 2009).

Na maioria dos trabalhos realizados em lagoas, áreas alagáveis e banhados, como constatado nos estudos de Irgang et al. (1984), Pott et al. (1989), Kita \& Souza (2003) e Matias et al. (2003), tal como no presente estudo, Cyperaceae tem se mantido em primeiro e Poaceae pelo menos entre o segundo ou terceiro lugar em relação à riqueza específica.

Espécies destas famílias, listadas no presente estudo, como Cyperus haspan, Cladium jamaicense, Cyperus luzulae, Eleocharis interstincta, Scleria hirtella, Scirpus giganteus, pertencentes a Cyperaceae e Briza calotheca, Dichanthelium sabulorum e Leersia hexandra, pertencentes a Poaceae, são de ampla distribuição em banhados do sul do Rio Grande do Sul, geralmente citadas nos estudos florísticos que abrangem esta região (Irgang et al. 1984; Cordazzo \& Seelinger 1988; Batista et al. 2007).

Por outro lado, Asteraceae, normalmente registrada nos estudos de ambientes aquáticos com baixa diversidade específica (Bove et al. 2003; Rocha et al. 2007), no presente estudo mostrou-se bem representativa. A contribuição desta família em termos de diversidade específica é incrementada especialmente pelas primeiras parcelas que representam área marginal do banhado, de topografia pouco mais elevada, visivelmente com menor umidade no substrato e adjacente a uma área de campo arenoso, onde muitas espécies coabitam nas duas áreas (banhado e campo).

Embora a maior parte das espécies de Asteraceae levantadas na área do presente estudo seja típica de ambientes úmidos, como Eclipta megapotamica, Senecio bonariensis (Irgang \& Gastal Jr. 1996), Enydra sessilis (Lima et al. 2006), algumas delas, como Achyrocline alata e Baccharis crispa, também ocorrem em áreas campestres (Ritter

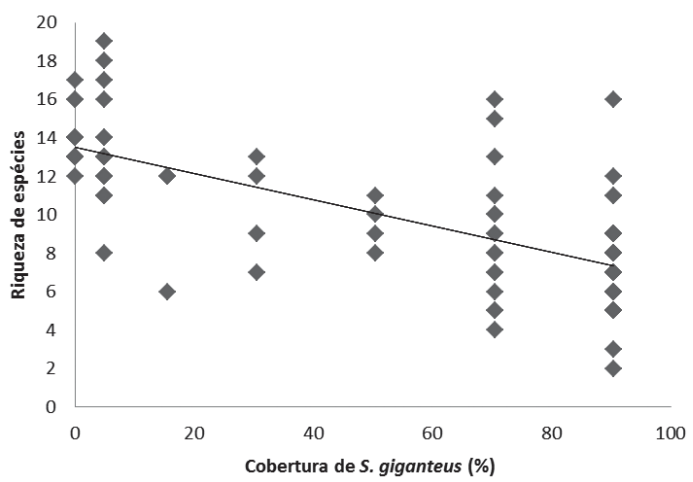

Figura 6 - Relação entre a cobertura de $S$. giganteus e a riqueza de espécies encontradas em cada unidade amostral pela regressão linear.

Figure 6 - Relationship between the coverage of $S$. giganteus and species richness found in each sampling unit using linear regression.

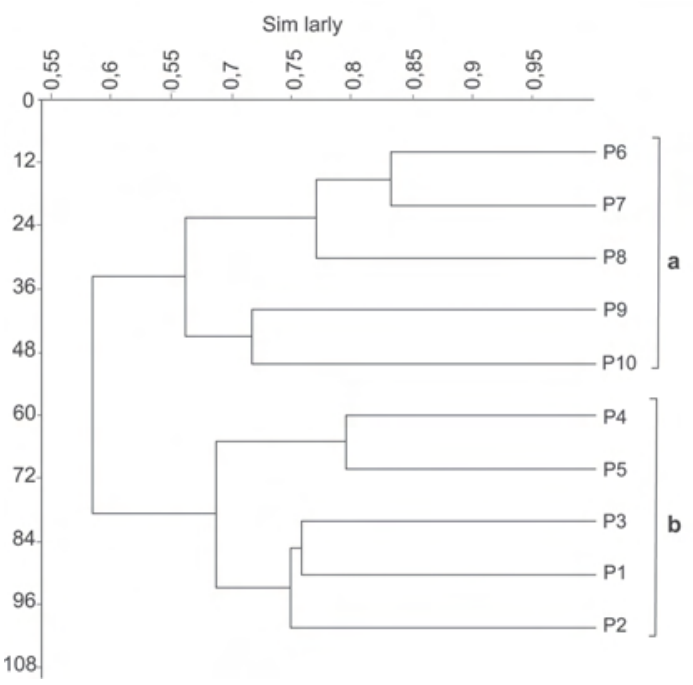

Figura 7-Dendrograma da analise de agrupamento entre as parcelas da margem em relação ao interior do banhado. Similaridade estimada pelo índice de Sorensen. a - parcelas do interior do banhado; $\mathrm{b}$ - parcelas da borda do banhado. Figure 7 - Dendrogram of cluster analysis of the margin between the plots in relation to the interior of the wetland. Similarity index estimated by Sorensen. a - portions of the interior of the wetland; $b$ - portions of the edge of the wetland.

\& Baptista 2005), assim incrementando a diversidade local. A riqueza de Asteraceae e Poaceae se justifica não somente pelo banhado possuir uma área de campo adjacente, mas também por serem famílias bem representadas no Rio Grande do Sul (Ritter \& Baptista 2005; Welker \& Longhi-Wagner 2007). 
$\mathrm{Na}$ área estudada, a maior dominância de cobertura pelas Cyperaceae está relacionada à presença de a $S$. giganteus, que ocupa quase $50 \%$ da cobertura total, ocorrendo desde áreas marginais até o interior do banhado, onde forma grupos densos e emergentes. Distribuição similar foi também observada por Kandus \& Adamoli (1993) em área do delta do Rio Paraná em Buenos Aires, que atribuíram a dominância desta espécie a alta densidade de folhas no dossel, uma ampla extensão de biomassa acima e abaixo do solo e por ser uma planta perene que apresenta vantagem competitiva devido a sua estratégia de reprodução por "dominância clonal", gerando uma diminuição significativa da diversidade.

De acordo com Bove et al. (2003), muitos ambientes sofrem alterações fitofisionômicas marcantes relacionadas com períodos de seca e de alagamento. Algumas espécies anuais desaparecem completamente na época de estiagem e outras, apesar de suportarem os períodos de seca, têm a população profundamente reduzida. Nos transectos levantados, no presente estudo, não foi observada alteração fitofisionômica marcante, ao ponto de alterar os percentuais de cobertura, permanecendo $S$. giganteus como dominante na maioria das parcelas, como também permaneceram dominantes as espécies que detinham maiores índices de cobertura em outras parcelas. Nestes casos as espécies anuais normalmente representaram baixos índices de cobertura e não influenciaram os índices de cobertura das espécies dominantes, independente da época do ano.

Algumas espécies comuns em ambientes úmidos como Leersia hexandra, Dichanthelium sabulorum, Hygrophila costata, foram também bastante frequentes na área de estudo, ocupando os estratos mais baixos da vegetação. Segundo Kozera et al. (2009), estas espécies podem inicialmente passar desapercebidas, por ficarem encobertas pelas espécies emergentes. No caso do presente estudo, visualmente, S. giganteus foi a espécie emergente que mais contribuiu para ocultar muitas espécies que colonizam estratos mais baixos.

Outra condição observada no presente estudo, possivelmente influenciada pela ampla extensão de biomassa da parte aérea de $S$. giganteus, foi a ausência de espécies flutuantes e submersas e ocorrência apenas de plantas enraizadas ao substrato, predominando formas anfíbias (94\%). A predominância desta forma biológica, também foi constatada nos estudos de Matias et al. (2003), Moura-Junior et al. (2009) e Spellmeier et al. (2009).
Segundo Thomaz (2002) um dos fatores limitantes para a presença de plantas submersas é a disponibilidade da luz que penetra na coluna d'água. Embora esta variável abiótica não tenha sido avaliada no presente estudo, verificou-se que a biomassa da parte aérea de $S$. giganteus prejudicou a penetração de luz e isso provavelmente afetou a colonização tanto de espécies aquáticas submersas, como também de flutuantes.

A composição da vegetação macrofítica pode ainda estar condicionada a outros atributos ambientais como o tamanho da área, a diversidade de habitats, disponibilidade e condutividade da água e a concentração de nutrientes (Rolon et al. 2010). No presente estudo, a análise de agrupamento mostrou uma separação em dois grupos, com diferença na composição florística das parcelas mais marginais em relação aquelas mais interiores do banhado, semelhante ao verificado no estudo de Matias et al. (2003), que observou a ocorrência de um zoneamento na vegetação conforme a espessura da lâmina de água, apresentando uma maior diversidade de espécies na área de interface entre $\mathrm{o}$ ambiente terrestre e o aquático.

Apesar da contribuição especial das espécies raras para a separação dos grupos, o grau de umidade do substrato, como já mencionado anteriormente, não foi mensurado, porém, visualmente parece contribuir para o agrupamento das parcelas em dois grupos. Percebeu-se que a maior parte das parcelas da margem do banhado ou próximas (grupo B) tem umidade reduzida e muitas espécies que colonizam o campo arenoso adjacente passam a compor esta comunidade. São típicas dessas formações campestres principalmente as espécies representantes de Asteraceae e Poaceae (Ritter \& Batista 2005; Welker $\&$ Longhi-Wagner 2007). Por outro lado, as parcelas que constituem o grupo A, visivelmente em substratos mais úmidos e alagados, apresentam espécies bastante típicas de banhados (Irgang \& Gastal Jr. 1996), especialmente as exclusivas deste grupo, conforme descrito acima, nos resultados.

O levantamento florístico e fitossociológico realizado no Banhado 25 revelou uma grande riqueza de espécies, com predomínio de Scirpus giganteus na cobertura, a qual caracteriza fitofisionomicamente a área estudada. Verificou-se que este alto índice de cobertura difere bastante dos índices encontrados para outros inventários realizados para a flora de ambientes alagados no estado. Sendo assim, estudos mais amplos, que levem em consideração fatores abióticos são necessários para subsidiar o entendimento da dominância desta espécie no local estudado, bem como sua relação com a composição florística da área. 


\section{Agradecimentos}

À FAPERGS, o financiamento de recursos para o desenvolvimento do projeto; ao ICB, o apoio logístico; aos colegas Leonardo Estima e Cínthia Almeida, o auxílio nos trabalhos de campo; ao Prof. Dr. Ubiratã Soares Jacobi, o auxilio profissional e as bibliografias indicadas; ao Eduardo L. Hettwer Giehl e as professoras Ana Maria Volkmer e Flávia Kneip, o auxilio nas análises estatísticas.

\section{Referências}

Amaral, M.C.E.; Bittrich, V.; Faria, A.D.; Anderson, L.O. \& Aona, L.Y.S. 2009. Guia de campo para plantas aquáticas e palustres do estado de São Paulo. Holos, São Paulo. 452p.

Amato, C.G.; Sponchiado, M. \& Schwarzbold, A. 2007. Estrutura de uma comunidade de macrófitas aquáticas em um açude de contenção (São Jerônimo, RS). Revista Brasileira de Biociências 5: 828-830.

APG III. 2009. An update of the Angiosperm Phylogeny Group classification for the orders and families of flowering plants: APG III. Botanical Journal of the Linnean Society 161:105-121.

Barbier, E.B.; Acreman, M. \& Knowler, D. 1997. Economic valuation of wetlands: a guide for policy makers and planners. Ramsar Convention Bureau; Department of Environmental Economics and Environmental Management; University of York; IUCN-the World Conservation Union, Cambridge. 135p.

Batista, T.L.; Canteiro, R.C.A.; Dorneles, L.P.P. \& Colares, I.G. 2007. Levantamento florístico das comunidades vegetais na Área de Proteção Ambiental da Lagoa Verde, Rio Grande, RS. Revista Brasileira de Biociências 5: 225-227.

Bertoluci, V.D.M.; Rolon, A.S. \& Maltchik, L. 2004. Diversidade de macrófitas aquáticas em áreas úmidas do município de São Leopoldo, Rio Grande do Sul. Pesquisas, série Botânica 54: 187-199.

Bove, C.P.; Gil, A.S.B.; Moreira, C.B. \& Anjos, R.F.B. 2003. Hidrófitas fanerogâmicas de ecossistemas aquáticos temporários da planície costeira do estado do Rio de Janeiro, Brasil. Acta Botanica Brasilica 17: 119-135.

Braun-Blanquet, J. 1979. Fitossociologia: bases para el estúdio de lãs comunidades vegetales. H. Blum e Ediciones, Madrid. 820p.

Burger, M.I. Situação e ações prioritárias para a conservação de Banhados e áreas úmidas da zona costeira. Disponível em <http://www.anp.gov.br/brasilrounds/ round7/round7/guias_r7/sismica_r7/refere/ Banhados.pdf.> Acesso em Ago 2010.

Cabrera, A.L. \& Fabris, H.A. 1948. Plantas acuaticas de la Provincia de Buenos Aires. Tomo v. 2. Publicaciones Tecnicas. 131p.
Carvalho, A.B.P. \& Ozório, C.P. 2007. Avaliação sobre os banhados do Rio Grande do Sul, Brasil. Revista de Ciências Ambientais 1: 83-95.

Cervi, A.C.; Bona, C.; Moço, M.C.C. \& Von Linsingen, L. 2009. Macrófita aquáticas do município de General Carneiro, Paraná, Brasil. Biota Neotropica 9: 1-8.

Cook, C.D.K. 1996. Aquatic plant book. SBP Academic Publishing, The Hague. 228p.

Cordazzo, C.V. \& Seeliger, U. 1988. Guia ilustrado da vegetação costeira no extremo sul do Brasil. Ed. FURG, Rio Grande. 107p.

Costa, C.S.B.; Irgang, B.E.; Peixoto, A.R. \& Marangoni, J.C. 2003. Composição florística das formações vegetais sobre uma turfeira topotrófica da planície costeira do Rio Grande do Sul, Brasil. Acta Botanica Brasilica 17: 203-212.

Esteves, F.A.1998. Fundamentos de limnologia. Interciência, Rio de Janeiro. 602p.

Forzza, R.C. et al. 2010. Lista de espécies da flora do Brasil. Disponível em <http://floradobrasil.jbrj. gov.br/2010/>. Acesso em Set 2010.

Getzner, M. 2002. Investigating public decisions about protecting wetlands. Journal of Environmental Management 64: 237-246.

Hammer, O.; Harper, D.A.T \& Ryan, P.D. 2001. PAST: Paleontological statistics software package for education and data analysis. Palaeontologia Eletronica 4: 1-9. Disponível em <http://folk.uio.no/ ohammer/past>. Acesso em Set 2010.

Holland, M.M.; Risser, P.G. \& Naiman, R.J. 1991. Ecotones: the role of landscape boundaries in the management and restoration of changing environment. Chapman \& Hall, New York. 144p

Irgang, B.E. \& Gastal Jr, C.V.S. 1996. Plantas aquáticas da planície costeira do Rio Grande do Sul. UFRGS, Porto Alegre. 290p.

Irgang, B.E.; Pedralli, G. \& Waechter, J.L. 1984. Macrófitas aquáticas da Estação Ecológica do Taim, Rio Grande do Sul, Brasil. Roeesleria 6: 935-404.

Kandus, E. \& Adamoli, J.M. 1993. Freshwater marsh vegetation response to flooding patterns in the lower delta of the Parana river. Wetlands Ecology and Management 2: 213-222.

Kita, K.K. \& Souza, M.C. 2003. Levantamento florístico e fitofisionomia da lagoa Figueira e seu entorno, planície alagável do alto rio Paraná, Porto Rico, estado do Paraná, Brasil. Acta Scientiarum: Biological Sciences 25: 145-155.

Kozera, C.; Kuniyoshi, Y.S.; Galvão, F. \& Curcio, G.R. 2009. Composição florística de uma mata pioneira com influência fluvial em Balsa Nova, Paraná, Brasil. Floresta 39: 309-322.

Krusche, N.; Saraiva, J.M.B \& Reboita, M.S. 2002. Normas climatológicas de 1991 a 2000 para Rio Grande, RS. Universidade Federal de Santa Maria. 84p.

Lisbôa, F.F. \& Gastal C.V.S. 2003. Levantamento das macrófitas aquáticas na beira do Lago Guaíba no 
município de Guaíba, RS/Brasil. Caderno de Pesquisa, série Biologia 15: 17-27.

Lima, F.P.; Schneider, A.A. \& Matzenbacher, N.I. 2006. Nota sobre a ocorrência de Enydra sessilis (SW.) DC. (Asteraceae - Heliantheae) para o estado do Rio Grande do Sul, Brasil. Pesquisas, série Botânica 57: 153-156.

Maltchik, L.; Rolon, A.S.; Guadagnin, D.L.; \& Stenert, C. 2004. Wetlands of Rio Grande do Sul, Brazil: a classification with emphasis on plant communities. Acta Limnologica Brasiliensia 16: 137-151.

Matias, L.Q.; Amado, E.R. \& Nunes, E.P. 2003. Macrófitas aquáticas da Lagoa de Jijoca de Jericoacoara, Ceará, Brasil. Acta Botanica Brasilica 17: 623-631.

Mauhs, J.; Marchioretto, M.S. \& Budke, J.C. 2008. Riqueza e biomassa de macrófitas aquáticas em área úmida na planície costeira do Rio Grande do Sul, Brasil. Pesquisas, série Botânica 57: 289-302.

Moura-Junior, E.D.; Silva, S.S.L.; Lima, L.F.; Lima, P.B.; Almeida Jr., E.B.; Pessoa, M.L.; Santos-Filho, F.S.; Medeiros, D.P.W.; Pimentel, R.M.M. \& Zickel, C.S. 2009. Diversidade de plantas aquáticas vasculares em açudes do parque estadual de Dois Irmãos (PEDI), Recife-PE. Revista de Geografia 26: 278-293.

Müeller-Dombois, D. \& Ellenberg, H. 1974. Aims and methods of vegetation ecology. John Wiley, New York. 547p.

Neiff, J.J. 1997. El régimen de pulsos em ríos y grandes humedales de Sudamérica. In: Malvarez, A.I. \& Kandus P. (eds.). Tópicos sobre grandes humedales sudamericanos. ORCYT-MAB (UNESCO), Montevideo. 106p.

Pivari, M.O.D.; Salimena, F.R.G.; Pott, V.J. \& Pott, A. 2008. Macrófitas aquáticas da lagoa Silvana, Vale do Rio Doce, Minas Gerais, Brasil. Iheringia, Série Botânica 63: 321-327.

Pott, V.J.; Bueno, N.C.; Pereira, R.A.C.; De Salis, S.M. \& Vieira, N.L. 1989. Distribuição de macrófitas aquáticas numa lagoa na fazenda Nhumirim, Nhecolândia, Pantanal, MS. Acta Botanica Brasilica 3: 153-168.
Ritter, M.R. \& Baptista, L.R. de M. 2005. Levantamento florístico da família Asteraceae na "Casa de Pedra" e áreas adjacentes, Bagé, Rio Grande do Sul. Iheringia, Série Botânica 60: 5-10.

Rocha, C.G.; Resende, U.M. \& Lugnani, J.S. 2007. Diversidade de macrófitas em Ambientes aquáticos do IPPAN na Fazenda Santa Emília, Aquidauana, MS. Revista Brasileira de Biociências 5: 456-458.

Rolon, A.S.; Homem, H.F. \& Maltchik, L. 2010. Aquatic macrophytes in natural and managed wetlands of Rio Grande do Sul State, Southern Brazil. Acta Limnologica Brasiliensia 22: 133-146.

Schwarzbold, A. \& Schäfer, A. 1984. Gênese e morfologia das lagoas costeiras do Rio Grande do Sul. Amazoniana 9: 87-104.

Seeliger, U.; Odebrecht, C. \& Castello, J.P. 1998. Os ecossistemas costeiro e marinho do extremo sul do Brasil. Ecoscientia, Rio Grande. 326p.

Spellmeier, J.; Périco, E. \& Freitas, E.M. 2009. Composição florística de um banhado no município de Estrela/Rio Grande do Sul. Pesquisas, série Botânica 60: 367-381.

Thomaz, S.M. 2002. Fatores ecológicos associados à colonização e ao desenvolvimento de macrófitas aquáticas e desafios de manejo. Planta Daninha 20: 21-34.

Thomaz, S.M. \& Bini, L.M. 2003. Análise crítica dos estudos sobre macrófitas aquáticas desenvolvidos no Brasil. In: Thomaz, S.M. \& Bini, L.M. (eds.). Ecologia e manejo de macrófitas aquáticas. EdUEM, Maringá. Pp. 19-38.

Thomaz, S.M.; Carvalho, P.; Padial, A.A. \& Kobayashi, J.T. 2009. Temporal and spatial patterns of aquatic macrophyte diversity in the Upper Paraná River floodplain. Brazilian Journal of Biology 69: 617-625.

Tropicos W3. 2010. MOBOT - Missouri Botanical Garden. Disponível em <http://www.mobot.org >. Acesso em Set 2010.

Welker, C.A.D. \& Longhi-Wagner, H.M. 2007. A família Poaceae no Morro Santana, Rio Grande do Sul, Brasil. Revista Brasileira de Biociências 5: 53-92. 